Y. Kitaoka

Nagoya Math. J.

Vol. 51 (1973), 79-89

\title{
TWO THEOREMS ON THE CLASS NUMBER OF POSITIVE DEFINITE QUADRATIC FORMS
}

\author{
YOSHIYUKI KITAOKA
}

0. In this note we study the estimate from above and below and the asymptotic behaviour of the class number of positive definite integral quadratic forms.

1. Let $S_{1}, S_{2}$ be positive definite matrices of degree $m$; then $S_{1}, S_{2}$ are called equivalent (resp. equivalent in the narrow sense) if $S_{1}={ }^{t} T S_{2} T$ for some $T$ in $G L(m, Z)$ (resp. $S L(m, Z)$ ). By definition $E(S)$ is the order of the unit group of $S$, i.e., the number of matrices in $G L(m, \boldsymbol{Z})$ such that ${ }^{t} T S T=S$. Let $m, D$ be natural numbers; by $H_{m}(D)\left(\right.$ resp. $\left.h_{m}(D)\right)$ we denote the number of equivalence classes (resp. equivalence classes in the narrow sense) in positive definite integral matrices of degree $m$ and determinant $D$.

THEOREM 1. Let $m$ be a natural number larger than 2 , and $\varepsilon$ be any positive number. Then we have

$$
c_{1}(m) D^{(m-1) / 2} \leq H_{m}(D) \leq c_{2}(m, \varepsilon) D^{(m-1) / 2+\varepsilon},
$$

where $c_{1}(m)$ is a positive constant depending on $m$, and $c_{2}(m, \varepsilon)$ is a positive constant depending on $m$ and $\varepsilon$. Moreover we can take 0 instead of $\varepsilon$ if we consider cases of square-free $D$.

COROLlary. For even $m$ we have

$$
h_{m}(D) \sim * 2 H_{m}(D) \quad \text { as } D \rightarrow \infty .
$$

THEOREM 2. Let $m$ be a natural number; then

$$
H_{m}(D) \sim 2 \sum \frac{1}{E(S)} \quad \text { as } D \rightarrow \infty,
$$

Received October 30, 1972.

Revised February 12, 1973.

*) $f(x) \sim g(x)$ as $x \rightarrow \infty$ means $\lim _{x \rightarrow \infty} \frac{f(x)}{g(x)}=1$ 
where $S$ runs over a set of representatives of different equivalence classes in positive definite integral matrices of degree $m$ and determinant $D$.

CoRollary. Let $m$ be an odd natural number. Then we have

$$
\lim _{\substack{D \rightarrow \infty \\ D: \text { odd } \\ \text { square-free }}} \frac{H_{m}(D)}{D^{(m-1) / 2}}=\pi^{-m(m+1) / 4} \prod_{k=1}^{m} \Gamma\left(\frac{k}{2}\right) \prod_{k=1}^{(m-1) / 2} \zeta(2 k),
$$

where $\zeta(s)$ is the Riemann zeta-function.

Remark. It is possible that we obtain the similar result to Theorem 2 for the number of classes in a genus on some assumptions (for example, on the assumption that $D$ is square-free).

2. LEMma 1. The number of groups of finite order in $G L(m, Z)$ is finite up to conjugacy.

Proof. Let $G$ be a group of finite order in $G L(m, Z)$, and $S$ be the positive definite matrix $\sum_{A \in G}{ }^{t} A A$. Then there exists an element $U$ in $G L(m, Z)$ such that ${ }^{t} U S U$ is reduced in the sense of Minkowski and the integral orthogonal group of ${ }^{t} U S U$ contains $U^{-1} G U$. From Satz 4 in [8], absolute values of all entries of $U^{-1} M U(M \in G)$ are not larger than some constant depending on $m$.

\section{Proof of Theorem 1 .}

Let $S$ be a positive definite integral matrix of degree $m$ and determinant $D$. Then the mass $M(S)$ of $S$ is by definition

$$
\sum \frac{1}{E\left(S_{k}\right)}
$$

where $S_{k}$ runs over the representatives of equivalence classes in the genus of $S$, and it is well known ([7])

$$
M(S)=\frac{2 \Gamma(1 / 2) \Gamma(2 / 2) \cdots \Gamma(m / 2)}{\pi^{m(m+1) / 4} \prod_{p} \alpha_{p}} \cdot D^{(m+1) / 2} \quad(m>1),
$$

where $\alpha_{p}=\alpha_{p}(S)$ is the density of $S$ at the prime $p$ and it is defined by

$$
\frac{1}{2} \lim _{\ell \rightarrow \infty}\left(p^{\ell}\right)^{-m(m-1) / 2} M\left(S ; p^{\ell}\right),
$$


where $M\left(S ; p^{\ell}\right)$ is the number of integral matrices $T \bmod p^{\ell}$ such that ${ }^{t} T S T \equiv S \bmod p^{\ell}$.

If $p$ does not divide $2 D$, then we have ([3], [7])

$$
\alpha_{p}= \begin{cases}\prod_{k=1}^{(m-1) / 2}\left(1-p^{-2 k}\right) & m: \text { odd } \\ \left(1-\left(\frac{(-1)^{m / 2} D}{p}\right) p^{-m / 2}\right) \prod_{k=1}^{(m / 2)-1}\left(1-p^{-2 k}\right) & m: \text { even } .\end{cases}
$$

If

$$
S \cong\left(\begin{array}{cc}
1_{m-2} & \\
& \varepsilon_{p} \\
& D \varepsilon_{p}^{-1}
\end{array}\right) \text { over } Z_{p} \text { for } p \mid D \text { and } p \neq 2
$$

where $\varepsilon_{p}$ is a unit of $Z_{p}$,

then we have ([3])

$$
\alpha_{p}=2 D^{(p)} \begin{cases}\left(1-\left(\frac{(-1)^{(m-1) / 2} \varepsilon_{p}}{p}\right) p^{-(m-1) / 2}\right) \prod_{k=1}^{(m-1) / 2-1}\left(1-p^{-2 k}\right) & m: \text { odd }, \\ \prod_{k=1}^{(m / 2)-1}\left(1-p^{-2 k}\right) & m: \text { even },\end{cases}
$$

where $D^{(p)}$ represents the $p$-part of $D$.

If $8 \mid D$, and

$$
S \cong\left(\begin{array}{ll}
A & \\
& D
\end{array}\right) \text { over } Z_{2}
$$

where $A$ is unimodular over $Z_{2}$ with determinant 1 , then by the similar proof to Hilfssatz 10, 11 in [3] we have

$$
M\left(S ; 2^{\ell}\right)=2^{\ell(m-1)} M\left(A ; 2^{\ell}\right) M\left(D ; 2^{\ell}\right),
$$

and so

$$
\alpha_{2}(S)=4 D^{(2)} \alpha_{2}(A),
$$

where $D^{(2)}$ represents the 2-part of $D$. Thus, on the assumption (2) if $8 \mid D$, we have

$$
\alpha_{2}(S) / D^{(2)} \leq c_{1}
$$

where $c_{1}$ depends on only $m$. From now on, $c_{i}$ represents a positive constant depending on only $m$, and $c_{i}(\varepsilon)$ depends on $m$ and $\varepsilon$. 
If $S$ satisfies the above condition (1) for any odd prime $p$, then we have

$$
\prod_{p \neq 2} \alpha_{p}^{-1}=\left\{\begin{array}{rr}
\frac{D^{(2)}}{D} \prod_{k=1}^{(m-1) / 2} \zeta(2 k) \prod_{k=1}^{(m-1) / 2}\left(1-2^{-2 k}\right) \prod_{\substack{p \mid D \\
p \neq 2}} 2^{-1}\left(1-p^{-(m-1)}\right) & \\
\quad \times\left(1-\left(\frac{(-1)^{(m-1) / 2} \varepsilon_{p}}{p}\right) p^{-(m-1) / 2}\right)^{-1} & m: \text { odd }, \\
\frac{D^{(2)}}{D} \frac{1}{\prod_{\substack{p \mid D \\
p \neq 2}} 2 \prod_{k=1}^{(m / 2)-1} \zeta(2 k) \cdot L\left(\frac{m}{2},\left(\frac{(-1)^{m / 2} D}{*}\right)\right) \prod_{k=1}^{(m / 2)-1}\left(1-2^{-2 k}\right)} \\
\times\left(1-\left(\frac{(-1)^{m / 2} D}{2}\right) 2^{-m / 2}\right) & m: \text { even . }
\end{array}\right.
$$

Thus on the assumptions (1), and (2) if $8 \mid D$, the mass $M(S)$ satisfies

$$
M(S) \geq c_{2} D^{(m-1) / 2} \prod_{\substack{p \mid D \\ p \neq 2}} 2^{-1} \begin{cases}\prod_{\substack{p \mid D \\ p \neq 2}}\left(1+\left(\frac{-\varepsilon_{p}}{p}\right) p^{-1}\right) & m=3, \\ 1 & m \geq 4 .\end{cases}
$$

Therefore if the number of odd primes dividing $D$ is zero or one, and $S$ satisfies above conditions (1) and (2) if $8 \mid D$ (for example, $\left.S=\left(\begin{array}{cc}1_{m-1} & \\ & D\end{array}\right)\right)$, then

$$
H_{m}(D) \geq M(S) \geq c_{3} D^{(m-1) / 2} \quad \text { for } m \geq 3 .
$$

Suppose that odd primes dividing $D$ are $p_{1}, p_{2}, \cdots, p_{t}(t \geq 2)$, and put the $p$-part of $D=p^{u_{p}}$. If there exists $j$ such that $u_{p_{j}}$ is odd, then for any given unit $\varepsilon_{p_{i}}$ of $Z_{p_{i}}(i \neq j)$ there exist a unit $\varepsilon_{p_{j}}$ of $Z_{p_{j}}$ and a positive definite integral matrix $S$ with $|S|=D$ such that $S$ satisfies the condition (1) and

$$
S \cong\left(\begin{array}{cc}
1_{m-1} & \\
& D
\end{array}\right) \quad \text { over } Z_{2}
$$

If any $u_{p_{i}}$ is even, then for any given unit $\varepsilon_{p_{i}}$ of $Z_{p_{i}}$ there exist a unit $\varepsilon_{2}$ of $Z_{2}$ and a positive definite integral matrix $S$ with $|S|=D$ such that $S$ satisfies the condition (1) and

$$
S \cong\left(\begin{array}{cccc}
1_{m-3} & & & \\
& \varepsilon_{2} & & \\
& & \varepsilon_{2}^{-1} & \\
& & & D
\end{array}\right) \text { over } \boldsymbol{Z}_{2} .
$$


Hence we obtain

$$
H_{m}(D) \geq \sum_{\substack{\varepsilon_{p_{i}} \\ p_{i} \\ i \neq j}= \pm 1} M(S) \geq \frac{1}{2} c_{2} D^{(m-1) / 2} \quad \text { for } m \geq 4
$$

and for $m=3$

$$
\begin{aligned}
H_{m}(D) & \geq \sum_{\substack{\varepsilon_{p_{i}} \\
p_{i} \\
i \neq j}} M(S) \geq c_{2} D 2^{-t} \sum \prod_{i=1}^{t}\left(1+\left(\frac{-\varepsilon_{p_{i}}}{p_{i}}\right) p_{i}^{-1}\right) \\
& \geq c_{2} D 2^{-t-1} \sum \prod_{\substack{i=1 \\
i \neq j}}^{t}\left(1+\left(\frac{-\varepsilon_{p_{i}}}{p_{i}}\right) p_{i}^{-1}\right) \\
= & 2^{-2} c_{2} D .
\end{aligned}
$$

Thus, we have proved $H_{m}(D) \geq c_{4} D^{(m-1) / 2}$.

Let $c_{5}$ be the maximal order of groups of finite order in $G L(m, Z)$. Then we have

$$
H_{m}(D) \leq c_{5} \sum M(S)
$$

where $S$ runs over the representatives of genera of positive definite integral matrices of degree $m$ and determinant $D$. This implies

$$
H_{m}(D) \leq c_{6} D^{(m+1) / 2} \prod_{p \nmid 2 D} \alpha_{p}^{-1} \prod_{p \mid 2 D}\left(\sum \alpha_{p}^{-1}\right),
$$

where $\sum \alpha_{p}^{-1}$ is the sum of the inverses of densities of matrices, up to equivalence, over $Z_{p}$ of degree $m$ and determinant $D$. On the other hand, we have

$$
\begin{aligned}
& \prod_{p \nmid 2 D} \alpha_{p}^{-1}= \begin{cases}\prod_{p \nmid 2 D} \prod_{k=1}^{(m-1) / 2}\left(1-p^{-2 k}\right)^{-1} & m: \text { odd }, \\
\prod_{p \nmid 2 D}\left(1-\left(\frac{(-1)^{m / 2} D}{p}\right) p^{-m / 2}\right)^{-1} \prod_{k=1}^{(m / 2)-1}\left(1-p^{-2 k}\right)^{-1} & m: \text { even },\end{cases} \\
& \leq c_{7} \text {. }
\end{aligned}
$$

Let

$$
S \cong\left(\begin{array}{llll}
p^{t_{1}} S_{1} & & \\
& \ddots & & \\
& & \ddots & \\
& & p^{t_{s}} S_{s}
\end{array}\right) \text { over } Z_{p}, \quad(p \neq 2)
$$


where $S_{i}$ are unimodular and $0 \leq t_{1}<t_{2}<\cdots<t_{s}$, and put $n_{i}=$ degree of $S_{i}, m_{i}=\sum_{k=i}^{s} n_{k}$. Then we get

$$
\alpha_{p}(S)=2^{s-1} p^{\omega\left(t_{i}, n_{i}\right)} \prod_{i=1}^{s} \alpha_{p}\left(S_{i}\right) \quad \text { for odd prime } p,
$$

where $\omega\left(t_{i}, n_{i}\right)=\sum_{k=1}^{s} t_{k} n_{k}\left(m_{k}-\left(n_{k}-1\right) / 2\right)$, and the sum $\sum \alpha_{p}^{-1}$ in (3) is

$$
\begin{aligned}
& \sum: \alpha_{p}^{-1}=\sum_{n_{k}, t_{k}} \sum_{\substack{\operatorname{deg}, S_{i}=n_{i} \\
i\left|S_{i}\right|=D / D(p)}} \alpha_{p}^{-1} \\
& =\sum_{n_{k}, t_{k}} \frac{2^{1-s}}{p^{\omega\left(t_{k}, n_{k}\right)}} \sum \prod_{k=1}^{s} \alpha_{p}\left(S_{k}\right)^{-1} \cdot
\end{aligned}
$$

We, now, estimate $\sum_{\hat{y}}^{s} \prod_{k=1}^{s} \alpha_{p}\left(S_{k}\right)^{-1}$ :

$$
\begin{aligned}
\sum \prod_{k=1}^{s} \alpha_{p}\left(S_{k}\right)^{-1} & \\
= & \sum \prod_{n_{k}=2} \alpha_{p}\left(S_{k}\right)^{-1} \prod_{n_{k} \neq 2} \alpha_{p}\left(S_{k}\right)^{-1} \\
= & \sum \prod_{n_{k}=2}\left(1-\left(\frac{-\left|S_{k}\right|}{p}\right) p^{-1}\right)^{-1} \prod_{n_{k} \neq 2} \alpha_{p}\left(S_{k}\right)^{-1} \\
= & \sum \prod_{n_{k}=2}\left(1+\left(\frac{-\left|S_{k}\right|}{p}\right) p^{-1}\right) \prod_{n_{k}=2}\left(1-p^{-2}\right)^{-1} \prod_{n_{k} \neq 2} \alpha_{p}\left(S_{k}\right)^{-1} \\
& \leq\left\{\prod_{k=2}^{m}\left(1-p^{-k}\right)^{-1}\right\}^{c 8} \sum \prod_{n_{k}=2}\left(1+\left(\frac{-\left|S_{k}\right|}{p}\right) p^{-1}\right) .
\end{aligned}
$$

If some $n_{k}$ is not 2 , then we can take any unit of $Z_{p}$ as $\left|S_{k}\right|$ for $k$ satisfying $n_{k}=2$, and $\sum \prod_{n_{k}=2}\left(1+\left(\frac{-\left|S_{k}\right|}{p}\right) p^{-1}\right)=2^{s-1}$. If all $n_{k}$ are 2 , then $\sum \prod_{k=1}^{s}\left(1+\left(\frac{-\left|S_{k}\right|}{p}\right) p^{-1}\right)=2^{s-1}\left(1+\left(\frac{(-1)^{m / 2} D / D^{(p)}}{p}\right) p^{-m / 2}\right)$. This implies

$$
\sum \alpha_{p}^{-1} \leq\left\{\prod_{k=2}^{m}\left(1-p^{-k}\right)^{-1}\right\}^{c_{9}} \sum_{n_{k}, t_{k}} \frac{1}{p^{\omega\left(t_{k}, n_{k}\right)}} \quad \text { for odd } p
$$

Put $D^{(p)}=p^{u_{p}}$, then $u_{p}=\sum n_{k} t_{k}$ and $\omega\left(t_{k}, n_{k}\right) \geq u_{p}$ and the equality arises if and only if $n_{1}=m-1, n_{2}=1, t_{1}=0$ and $t_{2}=u_{p}$.

If we confine ourselves to the case of square-free $D$, then we have $n_{1}=m-1, n_{2}=1, t_{1}=0$ and $t_{2}=u_{p}(=1)$. Hence in this case, we have

$$
\prod_{\substack{p \mid D \\ p \neq 2}} \sum \alpha_{p}^{-1} \leq c_{10} D^{(2)} / D
$$


We come back to the case of general $D$. Let $\beta_{s}$ be the number of partitions $m=\sum_{i=1}^{s} n_{i}, n_{i}>0$, and put $\ell=\omega\left(t_{k}, n_{k}\right)-u_{p}=t_{s} n_{s}\left(n_{s}-1\right) / 2$ $+\sum_{k=1}^{s-1} t_{k} n_{k}\left(m_{k}-\left(n_{k}+1\right) / 2\right)$; then in case of $s>1$, we have $t_{s-1} \leq \ell$ and $0 \leq t_{s-i} \leq \ell-i+1$. This implies that the number of systems $\left\{t_{k}\right\}_{k=1}^{s}$ such that $\ell=\omega\left(t_{k}, n_{k}\right)-u_{p}$ for some $n_{k}$ satisfying $\sum_{k=1}^{s} n_{k}=m, n_{k}>0$, $\sum n_{k} t_{k}=u_{p}$, and $0 \leq t_{1}<t_{2}<\cdots<t_{s}$ is at most $(\ell+1) \ell(\ell-1) \cdots$ $(\ell-s+3)$. Therefore we get

$$
\begin{aligned}
\sum_{n_{k}, t_{k}} p^{-\omega\left(t_{k}, n_{k}\right)} & \leq \frac{1}{D^{(p)}}\left\{\sum_{s=2}^{m} \beta_{s} \sum_{\ell=s-2}^{\infty} \frac{(\ell+1) \ell \cdots(\ell-s+3)}{p^{\ell}}\right\}+p^{-u_{p}(m+1) / 2} \\
& =\frac{1}{D^{(p)}}\left\{\sum_{s=2}^{m} \beta_{s} \frac{(s-1) !}{(p-1)^{s}} p^{2}+p^{-u_{p}(m-1) / 2}\right\},
\end{aligned}
$$

and finally we have

$$
\prod_{\substack{p \backslash D \\ p \neq 2}} \sum \alpha_{p}^{-1} \leq c_{10}(\varepsilon)\left(\frac{D^{(2)}}{D}\right)^{1-\epsilon}
$$

Now we estimate $\sum \alpha_{2}^{-1}$ :

Let $S \cong\left({ }^{S_{1}} S_{2}\right)$ over $Z_{2}$ and $S_{1}$ is unimodular of degree $n$ and $S_{2} \equiv 0(2)$; then from the similar proof of Hilfssatz 10, 11 in [3] it follows that

$$
M\left(S ; 2^{\ell}\right) \geq\left(2^{\ell-1}\right)^{(m-n) n} M\left(S_{1} ; 2^{\ell}\right) M\left(S_{2} ; 2^{\ell}\right)
$$

and so $\alpha_{2}(S) \geq 2^{1-(m-n) n} \alpha_{2}\left(S_{1}\right) \alpha_{2}\left(S_{2}\right)$. Let

$$
S \cong\left(\begin{array}{ccc}
2^{t_{1}} S_{1} & & \\
& \ddots & \\
& & \ddots \\
& & 2^{t_{s}} S_{s}
\end{array}\right) \text { over } Z_{2},
$$

where $S_{i}$ are unimodular and $0 \leq t_{1}<\cdots<t_{s}$ and put $n_{i}=$ degree of $S_{i}$ and $m_{i}=\sum_{k=i}^{s} n_{k}$; then we get

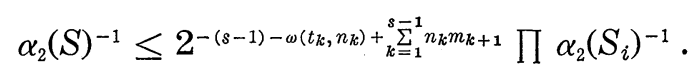

The number of unimodular matrices, up to equivalence, of degree $\leq m$, and the number of partitions $\sum_{i=1}^{s} n_{i}=m$, are finite, hence we get

$$
\begin{aligned}
\sum \alpha_{2}(S)^{-1} & \leq c_{11} \sum 2^{-\omega\left(t_{k}, n_{k}\right)} \\
& \leq c_{12} \frac{1}{D^{(2)}} .
\end{aligned}
$$


From these we have

$$
H_{m}(D) \leq c_{13}(\varepsilon) D^{(m-1) / 2+\varepsilon} .
$$

4. LemMa 2. Let $L$ be a positive definite quadratic lattice over $\boldsymbol{Z}$, and suppose that there is a non-trivial isometry $\sigma$ of $L$ such that $\sigma$ has 1 as an eigenvalue of $\sigma$. Then there exist non-zero two sublattices $L_{1}, L_{2}$ such that

$$
L \supset L_{1} \perp L_{2} \supset c_{14} L,
$$

where $c_{14}$ is a natural number depending on the rank of $L$.

Proof. Let $n$ be the order of $\sigma$. Then $n$ is not larger than some constant depending on the rank of $L$. The assumption implies $\sum_{i=1}^{n} \sigma^{i}$ $\neq 0$. Put $L_{0}=\{x \in L ; \sigma x=x\}$. Then $L_{0} \neq 0$, since there exists some $x$ in $L$ such that $\sum_{i=1}^{n} \sigma^{i} x \neq 0$, and the rank of $L_{0}$ is not equal to the rank of $L$. For any element $x$ in $L, \sum_{i=1}^{n} \sigma^{i} x$ is in $L_{0}$, and $n x-\sum_{i=1}^{n} \sigma^{i} x$ is in $L_{0}^{\perp}$. This means

$$
L \supset L_{0} \perp L_{0}^{\perp} \supset n L .
$$

Remark. $L \supset L_{1} \perp L_{2} \supset c_{14} L$ is equivalent to

$$
L_{1} \perp L_{2} \supset c_{14} L \supset c_{14}\left(L_{1} \perp L_{2}\right) \text {. }
$$

5. LEMMA 3. By $H_{m}^{0}(D)$ we denote the number of equivalence classes of positive definite integral matrices of degree $m$ and determinant $D$ which have a non-trivial unit with 1 as an eigenvalue. Then we have

$$
H_{m}^{0}(D) \leq c_{15}(\varepsilon) D^{(m-2) / 2+\varepsilon} \quad \text { for any } \varepsilon>0 .
$$

Proof. For $m=2, c_{16}(\varepsilon) D^{1 / 2-\varepsilon} \leq H_{2}(D) \leq c_{17}(\varepsilon) D^{1 / 2+\varepsilon}$ for any $\varepsilon>0$ is proved by Siegel. From Lemma 2 it follows

$$
\begin{aligned}
H_{m}^{0}(D) & \leq c_{14}^{m} \sum_{a=1}^{c_{14}^{m}} \sum_{b=1}^{[m / 2]} \sum_{c \mid a D} H_{b}(c) H_{m-b}(a D / c) \\
& \leq c_{18}(\varepsilon) \sum_{a=1}^{c_{12}^{2 m}[m / 2]} \sum_{b=1}^{[m}(a D)^{(m-b-1) / 2+\varepsilon} \sum_{c \mid a D} c^{(2 b \cdot m) / 2} \\
& \leq c_{19}(\varepsilon) \sum_{a=1}^{c_{11}^{2 m}} a^{(m-2) / 2+2 \varepsilon} D^{(m-2) / 2+2 \varepsilon} \\
& \leq c_{20}(\varepsilon) D^{(m-2) / 2+2 \varepsilon} .
\end{aligned}
$$

6. Proof of Corollary of Theorem 1.

Let $S$ be a positive definite integral matrix of even degree $m$ and 
determinant $D$. Suppose that any matrix which is equivalent to $S$ is always equivalent to $S$ in the narrow sense; then the unit group of $S$ contains a unit of whose determinant is -1 . This implies that the difference $2 H_{m}(D)-h_{m}(D)$ is at most the number of equivalence classes which have a unit of determinant -1 . From Lemma 3 and Theorem 1 follows our corollary.

\section{Proof of Theorem 2}

In case of $m=2$, let $S=\left(\begin{array}{ll}a & b \\ b & c\end{array}\right)$ and $D=a c-b^{2}$ and $c \geq a \geq 2|b|$. Since $E(S)>2$ implies $c=a$ or $a=|2 b|$, the number of equivalence classes which have a non-trivial unit is at most $c_{21}(\varepsilon) D^{\varepsilon}$ for any $\varepsilon>0$. This completes the proof in case of $m=2$. From Lemma 3 it is sufficient to prove Theorem 2 that we estimate the number of equivalence classes such that they have a non-trivial unit and any non-trivial unit has not 1 as an eigenvalue. Let $S$ be such a matrix, and $L$ be a lattice over $Z$ corresponding to $S$. We denote the orthogonal group of $L$ (= the unit group of $S$ ) by $G$. From the assumption, we see that $G$ contains a unit $\sigma$ such that $\sigma$ has not 1 as an eigenvalue and the order $q$ of $\sigma$ is an odd prime or 4 . If $q=4$, then $\sigma^{2}=-1$. If $q \neq 4$, then $\sigma+\cdots$ $+\sigma^{q}=0$. Hence the ring $Z[\sigma]$ is isomorphic to the maximal order $O$ of $Q(\sqrt[q]{1})$. Since, then, $L$ is a torsion-free $O$-module, from the theory of modules over Dedekind domain it follows that $L$ is $O$-isomorphic to a direct sum of ideals of $Q(\sqrt[q]{1})$ :

$$
L \cong A_{1} \oplus A_{2} \oplus \cdots \oplus A_{n},
$$

where $A_{1}=\cdots=A_{n-1}=O$, and the ideal $A_{n}$ is a (fixed) representative of some ideal class. (This ideal class is uniquely determined by L.) This identification transforms $S$ to a totally positive definite Hermitian matrix $H(S)=\left(h_{i j}\right)$ with $h_{i j}$ in $\left(A_{i} \bar{A}_{j} \theta\right)^{-1}$, where the bar denotes the complex conjugate and $\theta$ is the different of $Q(\sqrt[q]{1})$. Moreover if $S_{1}, S_{2}$ are equivalent and have $\sigma$ as a unit and $S_{1}=S_{2}[T]$ for some $T$ in $G L(m, Z)$ satisfying $\sigma T=\sigma T$, then for corresponding Hermitian forms $H\left(S_{1}\right), H\left(S_{2}\right)$ there exists a matrix $X=\left(x_{i j}\right)$ such that

$$
H\left(S_{1}\right)=X H\left(S_{2}\right)^{t} \bar{X} \text {, and } x_{i j}, x_{i j}^{\prime} \in A_{i}^{-1} A_{j},
$$

where $\left(x_{i j}^{\prime}\right)=X^{-1}$. We remark that there is a natural number $c$ such that all entries of $c H(S)$ are integers in $Q(\sqrt[q]{1})$, and the group $G=\{X$ 
$=\left(x_{i j}\right) ; x_{i j}, x_{i j}^{\prime} \in A_{i}^{-1} A_{j}$, where $\left.\left(x_{i j}^{\prime}\right)=X^{-1}\right\}$ and $G L(n, O)$ are commensurable. On the other hand, any totally positive definite Hermitian matrix is equivalent (with respect to $G L(n, O)$ ) to some element in $\bigcup_{i=1}^{d} S\left\{X_{i}\right\}$, where $S$ is a sufficiently large Siegel domain and $X_{i}$ is a non-singular integral matrix. ( $S, X_{i}, d$ depend on only $q$ and $n$.) This implies that the class number of positive definite Hermitian forms with the norm of determinant $\leq D$ is at most $c(q) D^{n / 2}$, where the constant $c(q)$ depends on only $q$. From these it follows that the number of equivalence classes in which there is some positive definite matrix $S$ such that $S$ has $\sigma$ as a unit and $|S| \leq D$ is at most $c_{22} D^{n / 2}$. Since $m>2$ implies $n<m-1$, we have proved Theorem 2.

\section{Proof of Corollary of Theorem 2.}

It is easy to calculate the mass of square-free and odd determinant by using [3], [6]:

$$
\begin{aligned}
\sum_{S} \frac{1}{E(S)}= & \frac{D^{(m-1) / 2}}{4 \pi^{m(m+1) / 4}} \prod_{k=1}^{m} \Gamma\left(\frac{k}{2}\right)^{(m-1) / 2} \prod_{k=1} \zeta(2 k) \\
& \times\left\{\left(1+2^{-(m-1) / 2}\right)\left(1+\delta\left(\frac{-1}{D}\right)^{\frac{m+1}{2}} D^{-(m-1) / 2}\right)\right. \\
& \left.+\left(1-2^{-(m-1) / 2}\right)\left(1-\delta\left(\frac{-1}{D}\right)^{\frac{m+1}{2}} D^{-(m-1) / 2}\right)\right\},
\end{aligned}
$$

where $S$ runs over a set of representatives of classes of positive definite integral matricies of odd degree $m \geq 3$ and of square-free and odd determinant $D$, and $\delta=(-1)^{(n+1)(n+2) / 2+((D-1) / 2) n}(n=(m-3) / 2)$. Corollary follows from this.

\section{REFERENCES}

[1] C. W. Curtis and I. Reiner, Representation theory of finite groups and associative algebras, Interscience Pub., 1962.

[2] M. Kneser, Klassenzahlen quadratischer Formen, Jahresbericht d. DMV, 61 (1958), 76-88.

[ 3 ] O. Körner, Die Maße der Geschlechter quadratischer Formen vom Range $\leq 3$ in quadratischen Zahlkörpern, Math. Ann., 193 (1971), 279-314.

[4] H. Minkowski, Diskontinuitätsbereich für arithmetische Äquivalenz, J. reine angew. Math., 129 (1905), 220-274.

[ 5 ] O. T. O'Meara, Introduction to quadratic forms, Springer-Verlag, 1963.

[6] H. Pfeuffer, Einklassige Geschlechter totalpositiver quadratischer Formen in totalreellen algebraischen Zahlkörpern, Jour. number theory 3 (1971), 371-411.

[ 7 ] C. L. Siegel, Über die analytische Theorie der quadratischen Formen, Ann. Math., 36 (1935), 527-606. 
[ 8 ] C. L. Siegel, Einheiten quadratischer Formen, Abh. Math. Sem. Univ. Hamburg, $13(1940), 209-239$.

[9] A. Weil, Discontinuous subgroups of classical groups, Lecture at the University of Chicago, 1958.

Nagoya University 